\title{
Using artificial neural network for labelling polygon features in topographic maps
}

\author{
Krzysztof Pokonieczny ${ }^{1}-$ Sylwia Borkowska ${ }^{1 凶}$ \\ ${ }^{1}$ Faculty of Civil Engineering and Geodesy, Military University of Technology in Warsaw, Poland

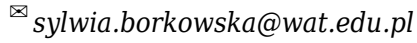

\begin{abstract}
The purpose of this article was to present the methodology which enables automatic map labelling. This topic is particularly important in the context of the ongoing research into the full automation of visualization process of spatial data stored in the currently used topographic databases (e.g. OpenStreetMap, Vector Map Level 2, etc.). To carry out this task, the artificial neural network (multilayer perceptron) was used. The Vector Map Level 2 was used as a test database. The data for neural network learning (the reference label localization) was obtained from the military topographic map at scale 1 : 50000 . In the article, the method of applying artificial neural networks to the map labelling is presented. Detailed research was carried out on the basis of labels from the feature class "built-up area". The results of the analyses revealed that it is possible to use the artificial intelligence computational methods to automate the process of placing labels on maps. The results showed that $65 \%$ of the labels were put on the topographic map in the same place as in the case of the labelling which was done manually by a cartographer. The obtained results can contribute both to the enhancement of the quality of cartographic visualization (e.g. in geoportals) and the partial elimination of the human factor in this process.
\end{abstract}

\section{Highlights for public administration, management and planning:}

- Map label placement is among key variables ensuring the usability of topographic maps across disciplines.

- We present the neural network approach for automating the process of labelling topographic maps with locality names.

- The presented case study applies to the military map in scale 1:50 000, but can be applied on other maps and geoportals.

\section{Introduction}

Labels are one of the most important elements of topographic map content. Using them together with map symbols distinguishes a map from spatial developments such as aerial photographs and orthophotomaps. An important task of the contemporary cartography is ensuring that labelling of any map, including a topographic map, is done properly and automatically to the greatest possible extent.

Research on automated label placement has been conducted for many years. In the paper (Freeman 2007), the author extensively presents prob-

\section{Keywords}

Artificial neural network Multilayer perceptron, Label localization, Topographic map

Received: 04 July 2019

Received in revised form: 31 October 2019

Accepted: 05 November 2019 lems and guides concerning automatic label placement. The full methodology for describing the process of labelling various objects on different maps have been presented in many articles, e.g. in the study (Reimer et al. 2015). It presents the method of placing text for objects created by groups of elements (e.g. archipelagos). The results were compared with labels put manually by a cartographer. (Wu et al. 2016) presents an algorithm which allows for determining the position of labels using the grid of squares superimposed on the map content. The presented methodology allows for labelling both line and area objects. In addition, it takes into account the mutual position of the la- 
bels, preventing their overlap. This problem is also included in the study (Kakoulis \& Tollis 2006). Harrie et al. (2005) raises the problem of automatic labelling on maps used in geoportals. The methodology presented by the authors allows to determine label placement in real time. A similar solution is presented in the paper (Been et al. 2006). A comprehensive approach to the discussed issues is presented in the article (Freeman 2005). This study describes the IT system which has been developed for 25 years and which performs automatic labelling on maps of various types and of different scales. Furthermore, (Rylov \& Reimer 2015) undertook to solve the problem of automatic label placement on topographic maps.

The main purpose of this article is to present the methodology for automating, to the highest possible extent, the process of labelling topographic maps with locality names. The main assumption was the fact that in most cases they are placed manually, usually by an experienced cartographer. The cartographer should take into account the rules of placing labels which are described in the instructions. However, in the case of Polish topographic maps, the instructions contain few guidelines which are very general ('Military Map at scale 1 : 50 000 - development and preparation for publication - instruction' 2011). The assumed aim of the research will be done by the artificial neural network, to which the experience and knowledge of the cartographer will be inputted through learning.

The application of the artificial neural network is justified by the fact that they are used for solving problems related to a large number of input data and in cases when an algorithm is unknown or difficult to implement (Suzuki 2013). The methodology also took into account land cover elements which are the content of the map.

\section{Methods}

\subsection{Input data}

The researches were carried out on the example of the location of the town labels on the Military Topographic Map at scale 1 : 50000 (M755 series). These maps are developed using the topographic database, which in the case of military maps is Vector Map Level 2 (VML2). Vector Map Level 2 (VML2) is a spatial database, standardized within NATO, with a level of detail corresponding to a topographic map at scale 1:50 000 ('Military specification MIL-V-89032 Vector Smart Map (VMAP) Level 2', 1993, p. 2). The method of data organization in VML2 is very precisely described in the DIGEST standard ('STANAG 7074, ed. 2: Digital Geographic Information Exchange Standard (DIGEST)' 1998). This development is a geographical database where data are divided into thematic categories, such as: boundaries, elevation, physiography, transport, land use, hydrography, vegetation, aviation content and industry. On the basis of VML2, the Military Topographic Map at scale 1:50 000, M755 series, is prepared. Digital Cartographic Model (DCM - the M755 series map) is thus created as a result of the cartographic visualization of this database (Fig. 1). In the cartographic production process, the automatic symbolisation of objects from VML2 is followed by the cartographic editing, which is predominantly done manually by an experienced cartographer. This process also includes the placement of the map labels. It should be emphasized that the majority of map development technologies (including topographic map development technologies) are based on similar assumptions,
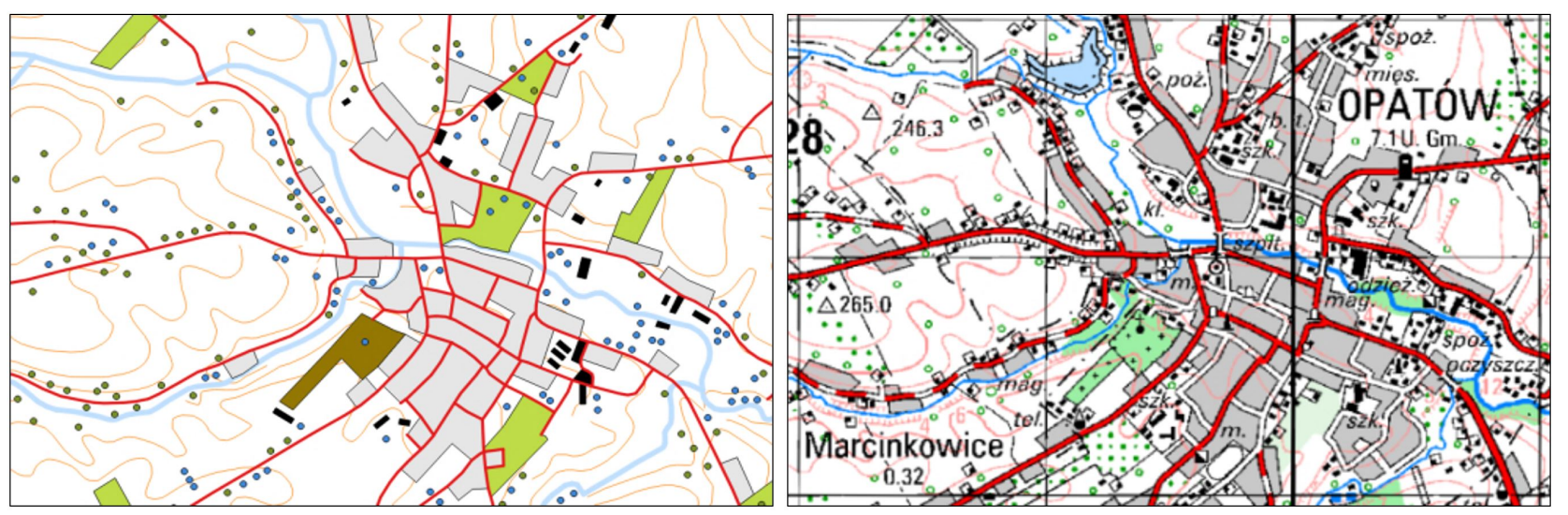

Fig. 1 An example of the visualization of the VML2 section (left side) and a section of the M755 series map (right side) 


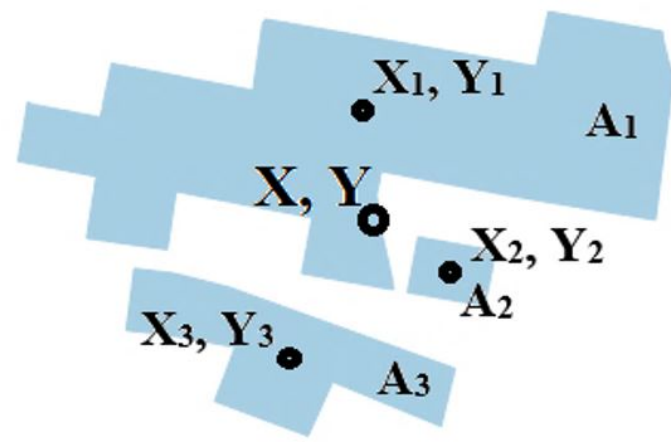

Fig. 2 An example of the centre of the rectangle.

which makes the presented methodology universal for polygon features in this study.

In order to present the application of the methodology, the localisation of the town and countryside labels located on 8 sheets of the map with map sheet identification numbers: N-34-121A,B, N-34-121-C,D, N-34-122-A,B and N-34-122C,D (sheets with 351 labels used for neural network learning) and N-34-123-A,B, N-34-123C,D, N-34-124-A,B and N-34-124-C,D (sheets used for the implementation of neural networks, 296 labels) was used.

\subsection{Construction of the data model}

The presented methodology assumes that the placement of labels will be considered for 9 locations, which are determined by rectangles surrounding the described object. , The class of "built-up area" objects, which is stored in the VMap Level 2 data model as a polygon feature, was selected as an example of the described methodology. The surrounding rectangles, for which the placement of labels was considered, were generated for each "built- up area" object. All 9 rectangles have the same shape and area. They were placed in a 3-by-3 grid, and their location is determined by the position of the central rectangle.

Tests were carried out for rectangles, during which they were automatically determined in four ways (Fig. 2):

1. The central rectangle is the MBR (Minimum Bounding Rectangle) of the built-up area.

2. The central rectangle is the MBR (Minimum Bounding Rectangle) of the built-up area whose center point has been moved

to a point with coordinates calculated as a weighted average according to the formula (1).

$$
X=\frac{\sum_{i=1}^{n}\left(X_{i} \cdot A_{i}\right)}{\sum_{i=1}^{n} A_{i}} ; Y=\frac{\sum_{i=1}^{n}\left(Y_{i} \cdot A_{i}\right)}{\sum_{i=1}^{n} A_{i}}
$$

Where: X, Y - coordinates of the designated point, $\mathrm{n}$ - number of objects included in a given built-up area, $X_{i} Y_{i}$ - centroid coordinates of individual parts of the built-up area, $A_{i}$ - area of individual parts of the built-up area

3. The central rectangle has the same area as the total built-up area. It was assumed that it has the same proportions as the MBR. Its center point is calculated as the average position of each centroid forming the built-up area.

4. The central rectangle has the same area as the total area of the built-up area. It was assumed that it has the same proportions as the MBR. Its center point is calculated as a weighted average according to the relation (1).

Due to the fact that the presented methodology takes into account the elements of the map content, for each rectangle in all variants, information about elements of land cover (which are located in the area of the surrounding rectangle) was obtained. This data was acquired from VMap Level 2 (Fig. 4):

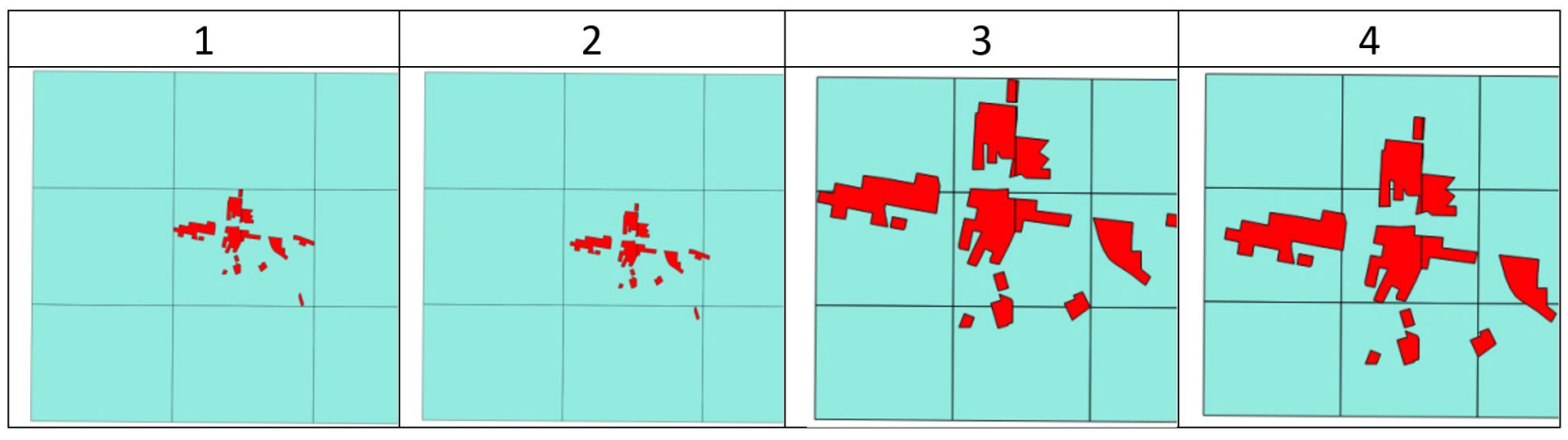

Fig. 3 An example of the determination of surrounding rectangles in four analysed variants. 


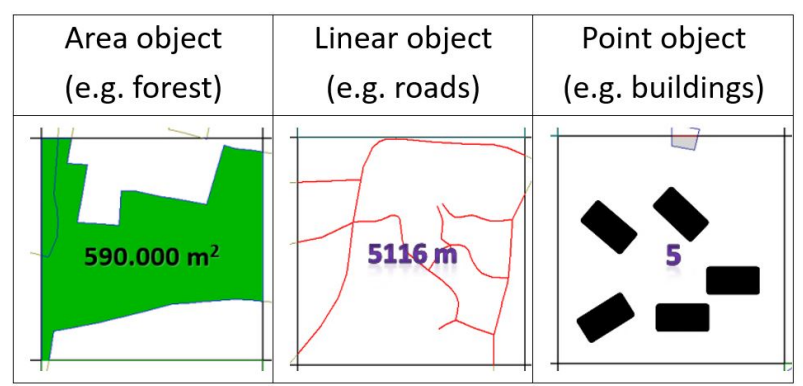

Fig. 4 An example of the visualization of data obtained from VML 2.

- for area objects (e.g. forests, lakes, built-up areas) - the surface of the area within a given rectangle;

- for linear objects (e.g. rivers, roads, railways, contour lines) - the length of a given linear object located in a specific rectangle;

- for point objects (buildings, farms) - the number of objects located in a rectangle.

As a result of analyses, the information about an area, length or quantity of 48 object classes from VMap Level 2 was obtained for each rectangle. In this way, the content of the vector database has been converted into the form of numerical parameters. This is necessary to input this data into the artificial neural network which can process signals only in the form of numerical values.

In addition to the data describing the terrain situation, it was necessary to collect data with the model distribution of labels. These data are obligatory for neural network learning. The data were acquired from the Military Topographic Map, where they were placed by a cartographer. In order to obtain them, the border of the label range was selected. Then, it was checked what area of this border overlaps with the individual rectangles (of the previously generated 9 rectangles grid, Fig. 5).
The final result of collecting input data for neural network learning was data set (collected separately for each of the generated surrounding rectangles) consisting of:

- 48 parameters acquired from VMap Level 2, representing elements of land cover (network inputs);

- surface of the area occupied by the envelope of the map label (network output) (Fig.5).

\subsection{Learning of artificial neural networks}

For the process of neural network learning, labels located on 4 sheets of the M755 series topographic map were used. 351 names of cities, towns and villages were located on the sheets (12 636 of surrounding rectangles were generated for them in 4 variants). For each configuration of surrounding rectangles, the above set of data has been inputted into the artificial neural network separately Due to the fact, that the values are collected in different units and numerical ranges, before the start of their processing by the artificial neural network, they were normalized to the range from 0 to 1 . The objects were normalized as described above Fig. 4.

In the researches, a feedforward neural network (called perceptron) consisting of three layers was tested. In the applied network, the supervised learning method with a teacher was used to determine the neuron weights in accordance with the backpropagation error method.

The values of weight are modified on the basis of input data (element of land cover) and the expected output of the neural network (area of envelope obtained from the map). In order to determine neuron weights, a supervised learning method with a teacher was used in accordance with the BFGS method (Broyden-Fletcher-Goldfarb-Shanno) with the SOS (Sum Of Squares) error function. The number of neurons in the hidden layers varies from 9

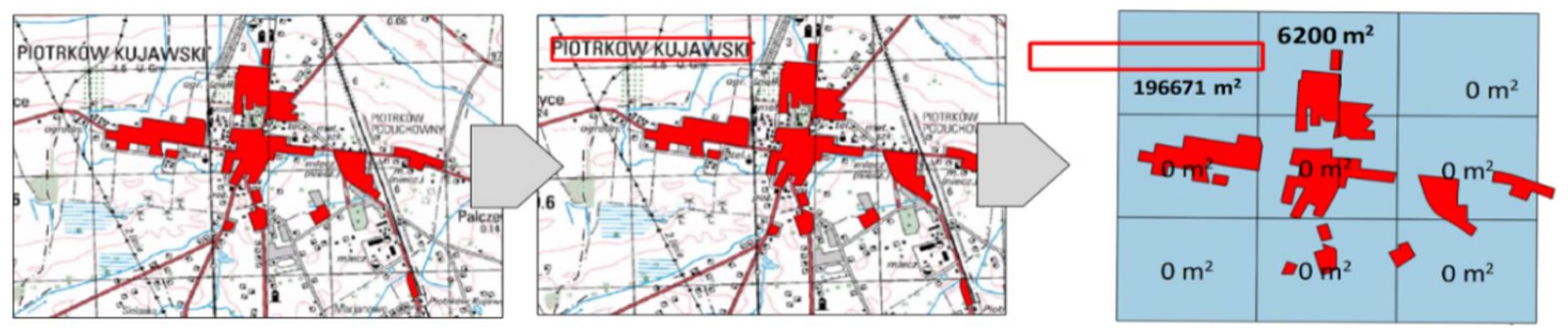

Fig. 5 A method of acquiring data for neural network learning 


\section{sciendo}
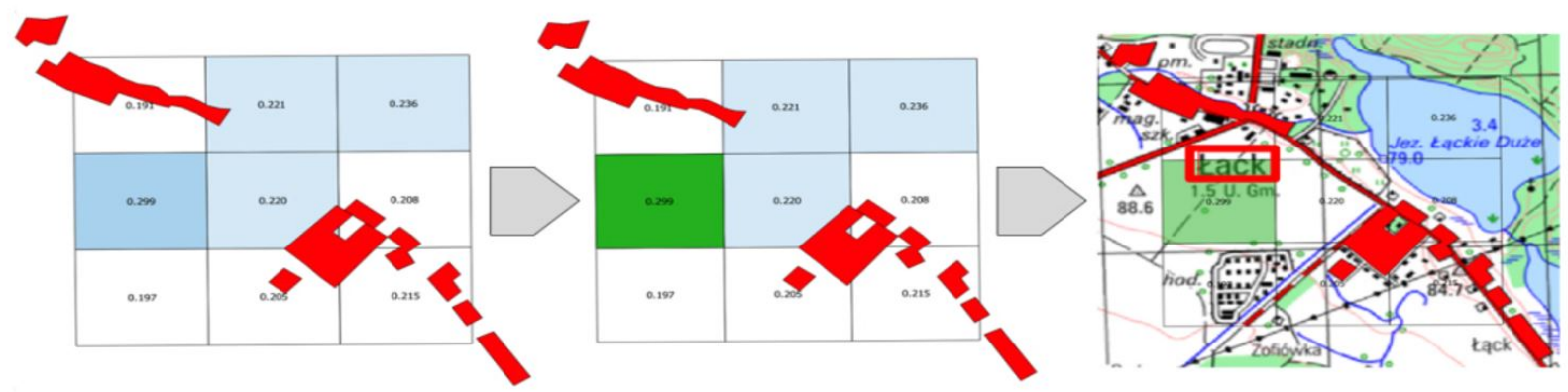

Fig. 6 The method of determining the labels' location
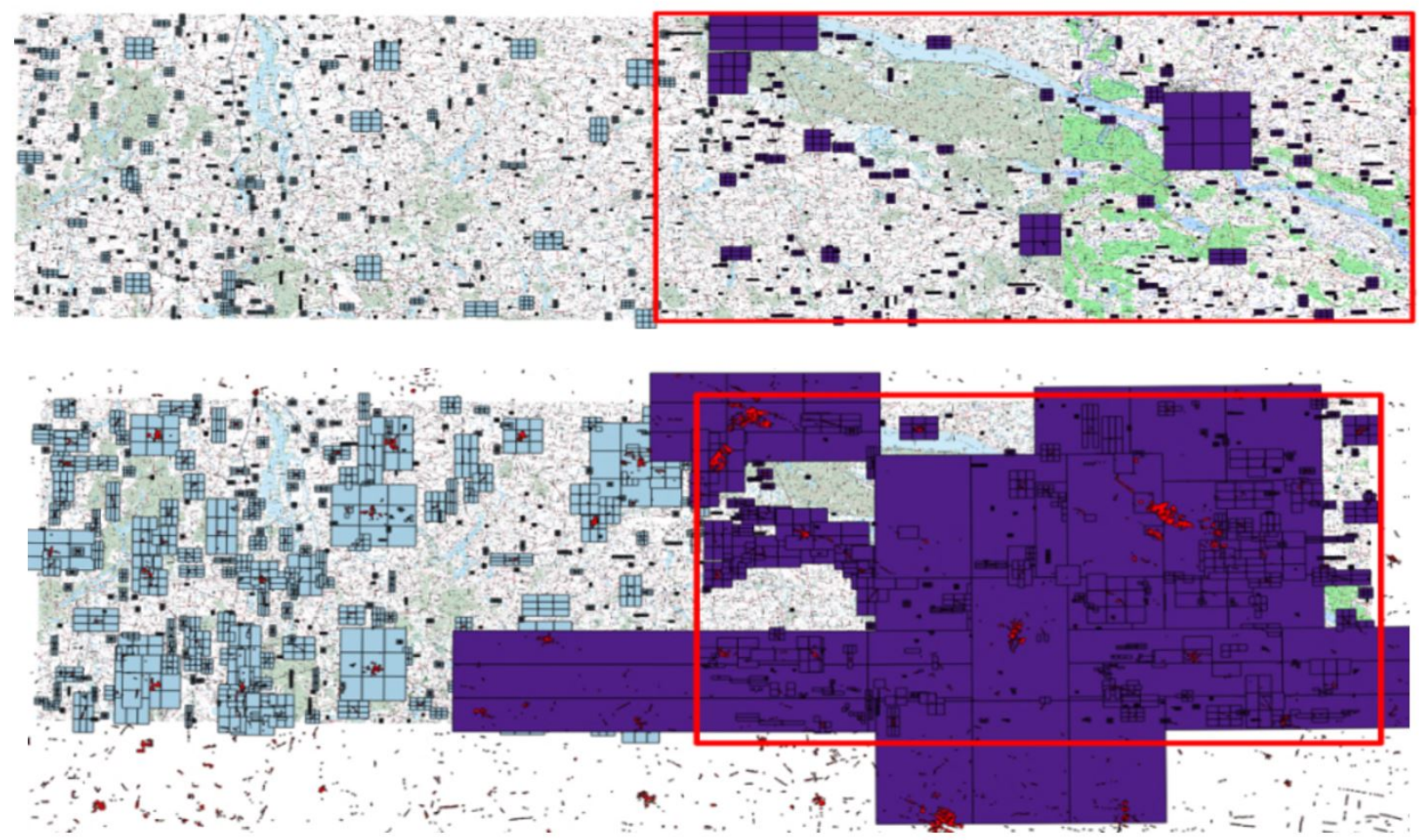

Fig. 7 The area used for network learning (on the left) and for implementing the neural network (right - red rectangle). On the top, the surrounding squares with an area equal to the the built-up area, below those with the area being MBR of the built-up area

to 89 . The number of training iterations varies from 4 to 49 .

Operations related to data processing in neural networks were carried out using the Statistica software version 12.5 ('STATISTICA Help | SANN Overviews - Network Training', n.d.). The proper functioning of the neural network is influenced mainly by the selection of its appropriate architecture (the activation functions used, the number of learning iterations, etc.). In order to find the optimal network architecture, a cross-validation was carried out. The learning data set has been divided into 3 subgroups: learning sample (70\% of samples) for learning the network, test sample (15\%), which is responsible for controlling the learning process and validation sample (15\%) used for final verification. The network "quality" was designated as the estimator determining the usefulness of the network for the label placement. It is the correlation coefficient between the output value and its prediction made by the neural network. The process of designation the optimal structure of neural net- 
work consists in generating a large number of neural networks (500 networks have been prepared in the analysis). Of these, 5 networks have been selected, for which the quality factor for the validation sample is the highest. To avoid the "overfitting" of neural network, the learning process was interrupted each time when the validation sample error increased. In the process of "searching" for the optimal neural network structure, the algorithm tested different numbers of neurons in the hidden layer and various activation functions (linear, logistic, hyperbolic tangent, exponential).

After completing the learning process of artificial neural networks, their operational use began. This implementation of the neural network consisted in entering the input data (terrain coverage parameters) to the neural network for a given rectangle. The result returned by the network on a single output neuron was a factor determining the suitability of placing the label in the rectangle. These coefficients were defined for all rectangles and the "winning" rectangle (the one, in which the label should be placed), was the rectangle with the highest coefficient (Fig. 6). The label was located as follows: the rectangle center was the label center. The research on the implementation of neural networks has been carried out in the area of four neighbouring (Fig. 7, right side) topographic map sheets. The idea was to verify, how the neural network works, when it is applied in areas (sheets of maps) different than those on which it was learned. Importantly, the map sheets on which the neural network was implemented, were developed by the same cartographer as the map sheets used for learning. This fact allows to suppose that the labels on these maps were placed using the same rules.

The final test was to compare the location of the labels generated by the artificial neural network with the real location of these labels on the map (i.e. placed by a cartographer).

\section{Results}

As a result of the "automatic network search" algorithm of the Statistica 12.5 software, 5 best neural networks (with the highest "quality" of the validation sample) were obtained. These networks were used to determine the location of labels for builtup area in the test area. The results were compared with the placement of labels on the map. They showed that in the case of rectangles based on the MBR of the built-up area (variants 1 and 2), the proposed algorithm located the labels in a similar way as a cartographer for $50 \%$ of the towns.
In $10 \%$ of cases, the labels were placed in a different way, but in accordance with cartographic rules. Better results were obtained for variants 3 and 4 because the neural network assigned $65 \%$ of the labels in the same way as the cartographer did, plus $15 \%$ were assigned correctly (from the cartographic point of view), but in different locations than it was on the map.

Fig. 8 shows the section of the map, where the actual location of the labels is marked with a green outline. The figure also shows the labels placed by the artificial neural network.

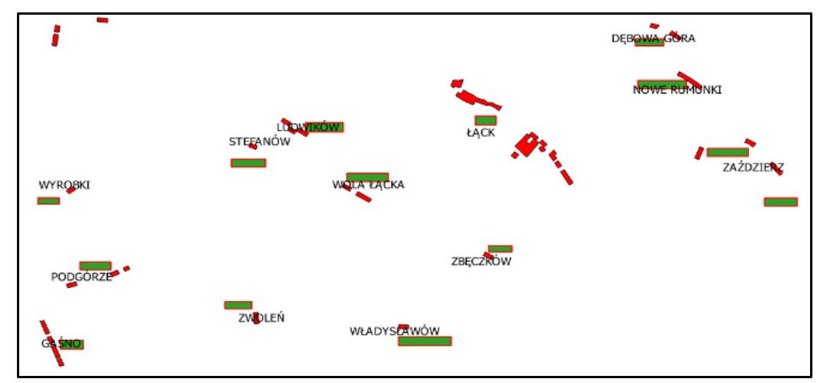

Fig. 8 A section of the map with a comparison of the labels placed by the neural network (names of localities) with the labels placed on the map by a cartographer (green border). The built-up areas are marked in red

\section{Discussion}

The obtained neural networks differ significantly in terms of architecture. It is noticeable that all the "best" networks obtained (those with the highest quality factor for the validation sample) have a different structure. This applies to the number of neurons in the hidden layer, the number of training iterations as well as the applied activation functions. This allows to conclude that we are not able to provide constant parameters of the neural network, which will carry out the task of label placement in the best way. Taking the above into account, the usage of the automatic neural network search algorithm is definitely a reasonable solution. Attention is drawn to the fact that the average quality of the validation sample for neural networks prepared for rectangles is the highest for variants based on the area of the built-up area (variant of surrounding rectangles no. 3 and 4 ), which is approx. 0.6 .In the case of primary fields of MBR shape, this coefficient is lower and amounts to about 0.5.

The implementation of the obtained neural networks which resulted directly in determining the lo- 
cation of labels for 4 neighboring topographical maps, and then comparing the obtained locations with the actual text placement on the topographic map, clearly shows that the results obtained for rectangles generated on the basis of the surface of built-up area are more appropriate for determining the location of the map labels. For these two configurations, about $65 \%$ of the labels were placed automatically in the same way as in the case when a cartographer did it manually. The reason for worse results for MBR-based configurations is the generation of very large rectangular envelopes because one locality (town) consists of many separate polygons, scattered over a large area (see Fig. 7, bottom right). It is also important that the influence of the center location of the rectangle on the obtained results is small (whether its center is determined as a weighted average or as an arithmetic average).

\section{Conclusions}

The obtained results clearly show that it is possible to automate the process of locating labels on the topographic map with the use of an artificial neural network. In the optimal configuration, $80 \%$ of the map labels have been placed correctly. On the other hand, when looking at this result, it should also be stated that every fifth label has been placed incorrectly, which excludes the possibility of using the presented methodology for the automatic placement labels on the map without any human participation. Taking this into account, the developed methodology was practically tested in an indirect way. In the first step, the labels were placed automatically by the neural network. Then, the cartographer reviewed them and made corrections. This process, even though it was not fully automatic, took about $50 \%$ less time than in the case of placing the labels manually.

Finally, it can be assumed that the aim of the article has been achieved. The methodology described in the article allowed the neural network to gather the knowledge and experience of the cartographer who placed the labels on the map manually. The neural network also took into account the surroundings of the placed labels, e.g. elements of topographic map content, collected in a topographic database.
Further research will be carried out towards increasing the number of possible locations of the labels. In its current form, the system considers the placement of the label for 9 locations. An increase in the number of surrounding rectangles will be considered, which is related to the computational capacity of the computer. In addition, further studies will be carried out to analyse the possibility of placing labels for non-area objects.

\section{References}

Been K, Daiches E, Yap C (2006) Dynamic Map Labeling. IEEE Transactions on Visualization and Computer Graphics 12(5): 773-780

Freeman H (2005) Automated cartographic text placement. Pattern Recognition Letters 26(3): 287-297.

Freeman $H$ (2007) On the problem of placing names on a map. Journal of Visual Languages \& Computing 18(5): 458-469.

Harrie L, Stigmar H, Koivula T, Lehto L (2005) An Algorithm for Icon Labelling on a Real-Time Map. In: Developments in Spatial Data Handling. Springer, Berlin, Heidelberg, pp. 493-507.

Kakoulis KG, Tollis IG (2006) Algorithms for the multiple label placement problem. Computational Geometry 35(3): 143-161.

Military Map in scale $1: 50$ 000, development and preparation for release - instruction, Wojskowa Mapa w skali 1 : 50000 opracowanie i przygotowanie do wydania - instrukcja. (2011). General Staff of Polish Armed Forces.

Military specification MIL-V-89032 Vector Smart Map (VMAP) Level 2. (1993). National Imagery and Spatial Agency.

Reimer A, Goethem A van, Rylov M, Kreveld M van, Speckmann B (2015) A formal approach to the automated labeling of groups of features. Cartography and Geographic Information Science 42(4): 333-344

Rylov MA, Reimer AW (2015) Improving label placement quality by considering basemap detail with a raster-based approach GeoInformatica 19(3): 463-486.

STANAG 7074, ed. 2: Digital Geographic Information Exchange Standard (DIGEST). (1998). Headquarters, Department of US Army, Washington.

STATISTICA Help | SANN Overviews - Network Training. (n.d.). Retrieved 10 August 2017, from http://documentation.statsoft.com/STATISTICAHelp.aspx?path=SANN/Overview/SANNOverviewsTrainingofNeuralNetowrks

Suzuki K (2013) Artificial Neural Networks - Architectures and Applications. InTech Open, Rijeka-London.

Wu C, Ding Y, Zhou X, Lu G (2016) A grid algorithm suitable for line and area feature label placement. Environmental Earth Sciences 75(20): 1368 